\title{
DESIGN AND CHARACTERIZATION OF PERIODICALLY CONDUCTIVE WOVEN FABRIC
}

\author{
Fuwang Guan', Zhaole Li' ${ }^{*}$, Zhuli Yang1, Chuyang Zhang ${ }^{1}$, Yiping Qiu ${ }^{1,2}$ \\ ${ }^{1}$ College of Textiles and Apparel, Quanzhou Normal University, Fujian 362000, China \\ ${ }^{2}$ College of Textiles, Donghua University, Shanghai 201620, China \\ *Corresponding author e-mail: lizhaole@163.com
}

\begin{abstract}
:
In this paper, a novel kind of electromagnetic (EM) functional textiles is proposed, which show high-pass characteristics as they interact with EM waves. The periodically conductive woven fabric was designed, fabricated, and measured. Specifically, by means of unit cell model building and EM simulation, the theoretical $S_{21}$ (transmission coefficient) and $S_{11}$ (reflection coefficient) curves were obtained. A concrete sample was fabricated through weaving process, and its transmission characteristics were measured in the microwave anechoic chamber. The measured and simulated results were highly consistent, demonstrating the validity of design process. Compared with the aluminum foil paper sample, the $S_{21}$ values of fabricated sample were a little smaller, and the reason could be attributed to yarn crimp and surface roughness. The EM characteristics of fabricated sample under two different polarization modes were slightly different, which was due to the beating-up tension of weaving process. The work could offer new research ideas, and the related products have potential advantages over rigid plates on the account of textile characteristics.
\end{abstract}

\section{Keywords:}

Electromagnetic functional fabric; frequency selective surface; high-pass characteristics; textile modeling; electromagnetic simulation

\section{Introduction}

Frequency selective surfaces (FSSs) have been widely studied over the past few decades, which were mainly focused on the design and characterization of new structures, active FSS, and curved or three-dimensional (3D) structures [1-4]. Traditionally, software simulation and numerical calculation methods were used to predict the transmission characteristics and design the corresponding array structure $[5,6]$. The involved fabrication methods were mainly printed circuit board (PCB) technology or other metallic coating and cutting techniques [7, 8], and therefore the final products were mainly rigid plates and composites, rarely involving soft materials.

Traditional electromagnetic (EM) shielding fabrics were fabricated through conductive polymer coating or conductive yarn-weaving process, which certainly degraded the wearing properties [9-11]. The novel fabrics with good shielding effectiveness (SE) as well as wearability were studied based on the concept of traditional FSSs, and the methods such as silk screen printing, computer embroidery technology, and cloth hot stamping process were used to obtain the periodic conductive pattern on the surface of fabrics [12,13]. The uninvolved textileforming technology, such as weaving, knitting, and basketry processes, could also be used to fabricate the products, in which the periodic conductive patterns are embedded in the fabrics, which is different from the form of metallized finishing, in which fabrics act as substrates. Actually, some researchers carried out the related work, but the work did not involve the specialized model and offer definite and effective design ideas $[14,15]$.
In this paper, the novel periodically conductive woven fabric is proposed. The unit cell model containing the information of textiles was built in TexGen and the full-wave simulation of the proposed structure was performed in CST Microwave Studio. Then, the sample with the corresponding conductive grid structure was woven and further tested in the anechoic chamber. On the whole, the paper offered a novel perspective and research idea for the FSS and EM functional textile researchers. The model building and software simulation work could effectively predict the transmission characteristics and help explore the influence rules of various factors. The weaving process could be a useful way in the product development. The obtained products have advantages over rigid materials in lightweight, softness, and low bending rigidity, which make them popular and irreplaceable in certain fields.

\section{Modeling and simulation process}

\subsection{Structure of periodically conductive woven fabric}

For most woven fabrics, warp yarns and weft yarns are mutually perpendicular. Based on this feature, woven frequency selective fabrics could be easily fabricated through periodic interweaving of conductive yarns and dielectric yarns. Although the position relation of warp and weft yarns is limited, the unit cell shapes could be different, because the number of conductive yarns could be adjusted freely. Figure 1 shows the schematic of three kinds of periodically conductive woven fabrics, in which the colored yarns are conductive and the gray yarns are dielectric. As shown in Figure 1, the corresponding unit cells could be 
extracted based on the periodic arrangement of conductive and dielectric yarns, as marked using red dashed square frame.

Periodically conductive woven fabrics with two-dimensional (2D), one-dimensional (1D), and combined unit cells are shown in Figure $1(\mathrm{a}-\mathrm{c})$, respectively, and they could represent almost all the orthogonal fabrics. By extracting the structure and material parameters of unit cells, such as conductivity of conductive yarns, dielectric properties of dielectric yarns, ratio of yarn arrangement number, yarn width and spacing, and fabric thickness, the equivalent models could be built to characterize the key features of periodically conductive woven fabrics, which would provide the basis for effective simulation process.

\subsection{Model building}

To simulate the transmission characteristics of EM waves precisely and efficiently, suitable unit cell models should be built, taking into account the practical situation as well as necessary assumptions. As a typical instance, the unit cell model was built as shown in Figures 1(a) and 2.

In Figure 2, the related information and dimensional parameters are clearly marked, where $w$ refers to the width of yarns, $g$ is the gap between two adjacent yarns, and $t$ represents the fabric thickness. The proposed model consists of 16 nonconductive yarns and two conductive yarns in both directions. In the paper, the plain fabric with the warp and weft thread density of $240 / 10 \mathrm{~cm}$ was selected on the basis to determine the parameters' values, and therefore the values of $w$ and $g$ were roughly determined as $0.45 \mathrm{~mm}$ and $0.1 \mathrm{~mm}$, respectively. The value of $t$ was assumed to be $0.1 \mathrm{~mm}$ considering the real thickness of fabricated sample. The model was built in TexGen software and exported for the subsequent simulation process. All the other unit cell models of proposed fabrics could be built using similar methods.

\subsection{Simulation settings and results}

The full-wave simulation was carried out in CST Microwave Studio. The periodic boundary condition (PBC) as well as Floquet port was employed. The sweep frequency range was $2-18 \mathrm{GHz}$ with the step of $0.032 \mathrm{GHz}$, and the adaptive tetrahedral mesh refinement was used. The conductivity and permittivity of two kinds of yarns in the model were set to be $6.1^{1} 10^{7} \mathrm{~S} / \mathrm{m}$ (equal to metallic silver) and 1.1 (equivalent value), respectively. In this paper, the transmission direction of EM waves is perpendicular to the surface of periodically conductive woven fabrics and of course the incidence angle could be adjusted to explore the angle stability. For the proposed model of periodically conductive woven fabric, the array characteristics are completely identical in two perpendicular directions, and therefore it is polarization insensitive. Figure 3 shows the simulated $S_{21}$ (transmission coefficient) and $S_{11}$ (reflection coefficient) curves under transverse electric (TE) as well as transverse magnetic (TM) mode, which present opposite variation tendency as expected.

As shown in Figure 3, the critical frequency point is $3.5 \mathrm{GHz}$ for both curves as the threshold values are set as -10 and $-0.5 \mathrm{~dB}$ severally, meaning that over $90 \%$ of EM waves could be shielded below $3.5 \mathrm{GHz}$. As the frequency of EM waves increases, more EM waves would traverse the proposed fabric and nearly $90 \%$ of EM waves could pass through the designed fabric near $18 \mathrm{GHz}$, showing obvious high-pass

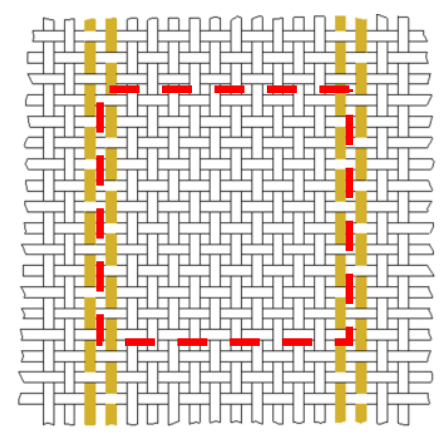

(b)

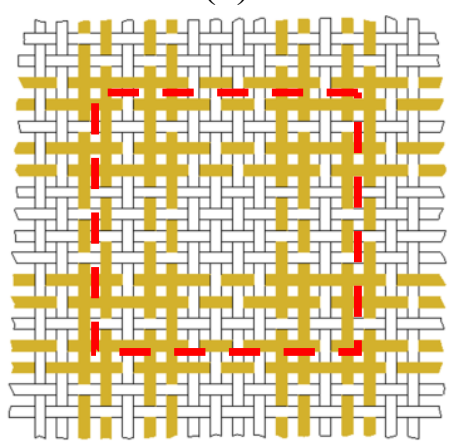

(c)

Figure 1. Three kinds of periodically conductive woven fabrics: a) 2D; b) 1D; c) combined Abbreviations: 1D, one-dimensional; 2D, twodimensional 


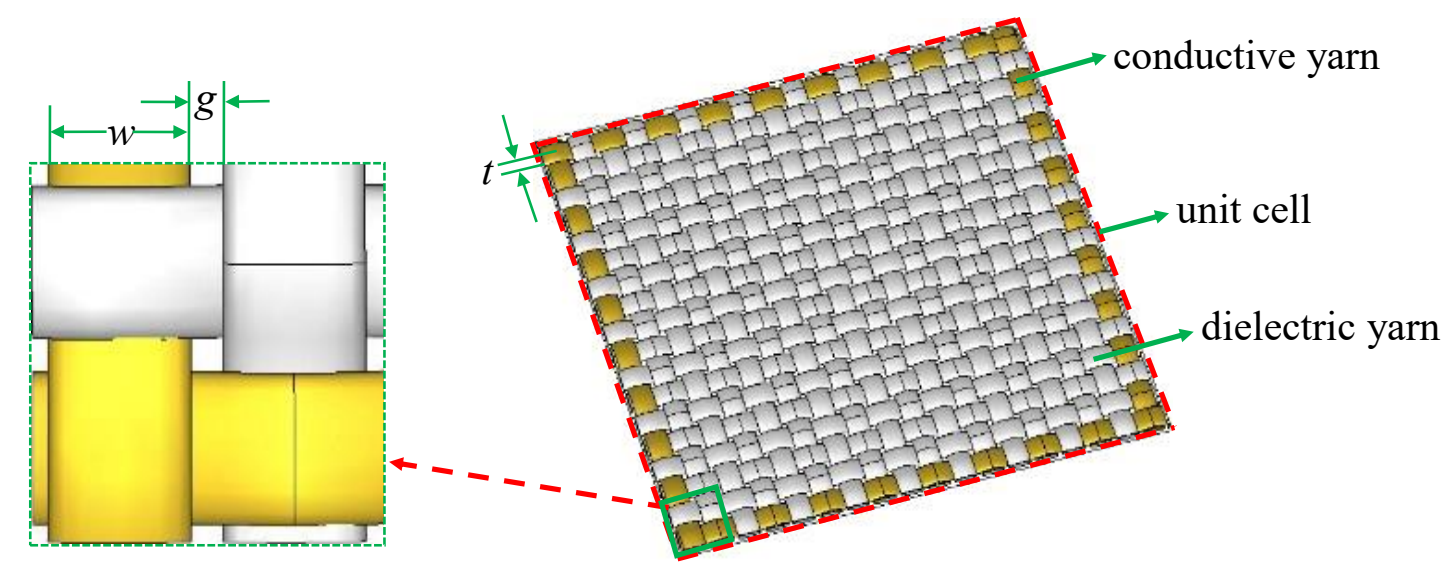

Figure 2. Unit cell model of $2 \mathrm{D}$ periodically conductive woven fabric Abbreviation: 2D, two-dimensional

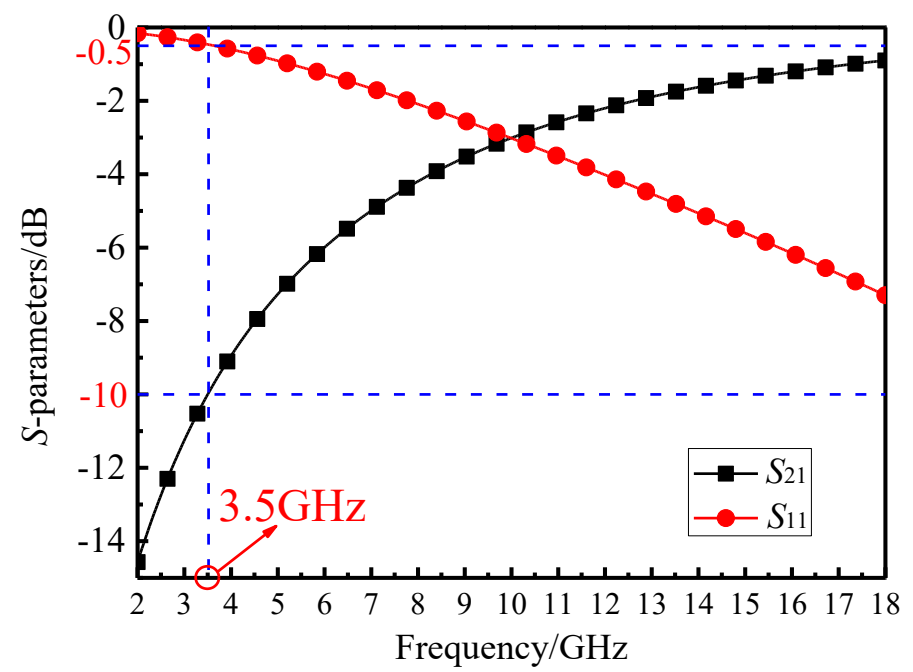

Figure 3. Simulated $S_{21}$ and $S_{11}$ curves at normal wave incidence Abbreviations: $S_{11}$, reflection coefficient; $S_{21}$, transmission coefficient

characteristics. Considering the actual processing errors, the array characteristics of warp and weft directions are not completely identical, and the influence of polarization mode would be discussed in the subsequent analysis process.

\section{Sample preparation and measurement}

Based on the simulation design, the corresponding sample was woven using the SGA598 semiautomatic loom as shown in Figure 4. Consistent with the simulation process, the fabric was plain weave with the thread density of $240 / 10 \mathrm{~cm}$. The silver-plated polyamide was adopted as the conductive yarn in the experiment. The cotton/polyamide (70/30) blended yarn was adopted as the nonconductive yarns, and the fineness was $50 \mathrm{~s}$. By controlling the ends per dent and beating-up tension, the sample with the accurate weaving density and yarn arrangement were obtained. The sample picture and partial enlarged detail are also shown in Figure 4, from which we could see that it has typical conductive grid structure and the thickness is measured to be roughly $0.1 \mathrm{~mm}$.

The testing process was performed in a microwave anechoic chamber at Nanjing University of Aeronautics and Astronautics using transmission method. The testing system is shown in Figure 5, in which the two horn antennas Tx and Rx (2$18 \mathrm{GHz}$ ) are, respectively, used for transmitting signals and receiving signals and connected with an Agilent N5230C vector network analyzer. The absorbing wall is removable and made of pyramidal construction units, and the sample with the square size of $300 \mathrm{~mm}$ ' $300 \mathrm{~mm}$ could be placed in the middle to test the transmission performance. As the horn antennas are placed as shown in Figure 5, the polarization mode is TM mode, and the TE mode could easily be realized by rotating the horn antennas $90^{\circ}$.

To eliminate the influence of background noise, two steps for the calibration were implemented. The transmission coefficient $T_{0}$ (in $\mathrm{dB}$ ) was first measured without sample, and then the transmission coefficient $T_{1}$ (in $\mathrm{dB}$ ) was measured with fabric sample. The actual transmission characteristic $T$ (in $\mathrm{dB}$ ) could be calculated by $T=T_{1}-T_{0}$.

\section{Results and discussion}

\subsection{Comparison of results}

To verify the validity of simulation process, the simulation and experimental results of periodically conductive woven fabric 


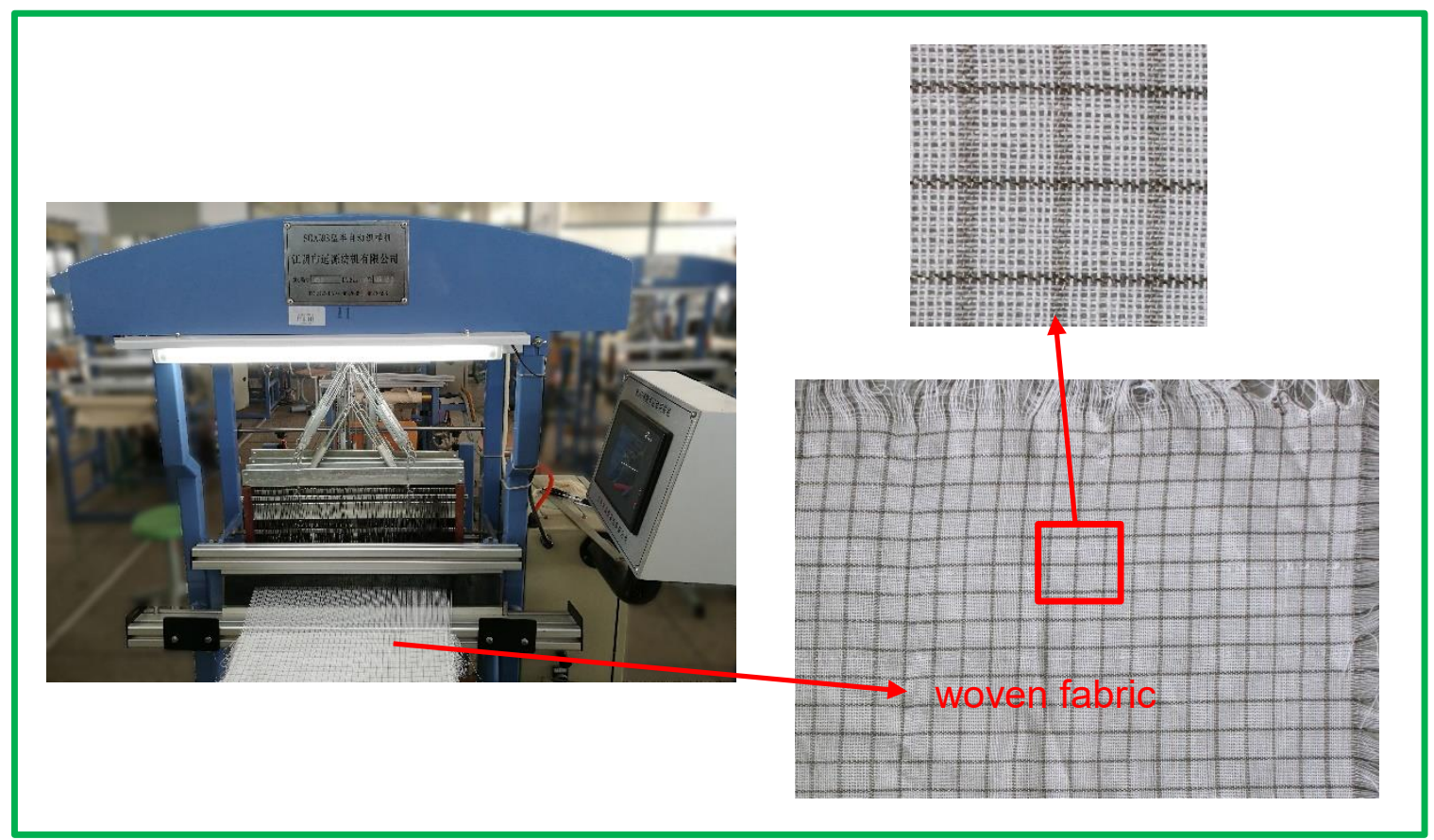

Figure 4. Weaving process and periodically conductive woven fabric

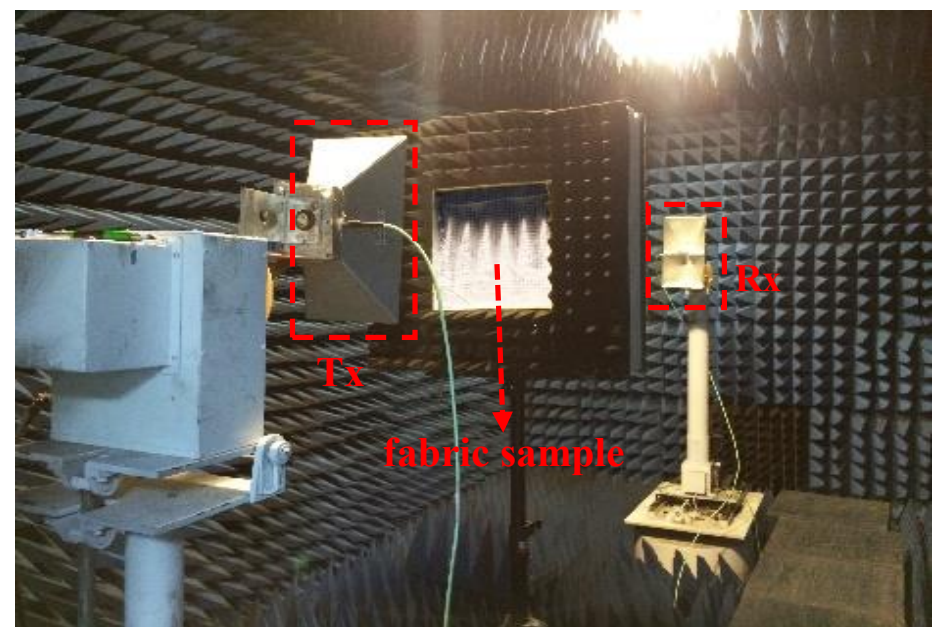

Figure 5. Measurement setup in the microwave anechoic chamber

were compared as shown in Figure 6. The conductive yarn fineness and conductivity in the measured sample were found to be $140 \mathrm{D}$ and $3.55 \Omega / \mathrm{cm}$, respectively. To evaluate its highpass characteristics more objectively, the aluminum foil paper sample with the same grid structure was prepared through the computer-based carving process and used for comparison. The involved method and materials were presented in the previous paper [16] and are not repeated in this paper. For comparative purpose, the polarization mode of EM waves was TE mode in the measurement as well as simulation process, and the influence of polarization mode is discussed in the following section.

On the whole, the measured and simulated results of proposed fabric show the same variation trend and have good consistency, and the small fluctuations and differences between the two curves could be attributed to the fact that the two kinds of yarns in the sample are actually different and the weft density may not be completely accurate as designed. In addition, the conductivity is not necessarily identical, because the yarn cross-section is estimated. However, the high data coincidence illustrates that all the approximations and assumptions are reasonable and effective.

Specifically, the measured $S_{21}$ values are a little smaller compared with the aluminum foil paper sample, and the biggest difference value reaches $2.4 \mathrm{~dB}$ (-5.3 and $-7.7 \mathrm{~dB}$, respectively), as marked in the figure. Considering the basically same structure parameters and similar material EM properties of two samples, the regular deviation should be attributed to the special fabric structure. Figure 7 shows the unit cell comparison of two kinds of high-pass FSSs.

Existing studies indicate that the equivalent inductors of grid structure play the decisive role in the macroscopic transmission characteristics, and the inductance values are positively correlated with the length of conductive wires $[17,18]$. Although the projected lengths of conductive wires 


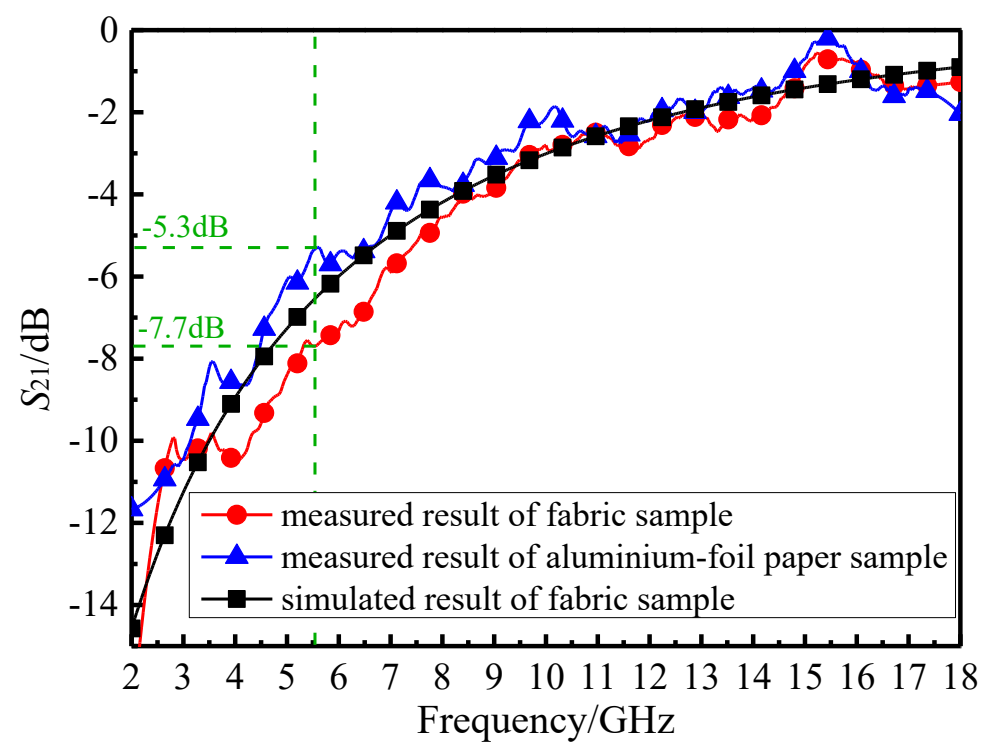

Figure 6. Comparison of measured and simulated results

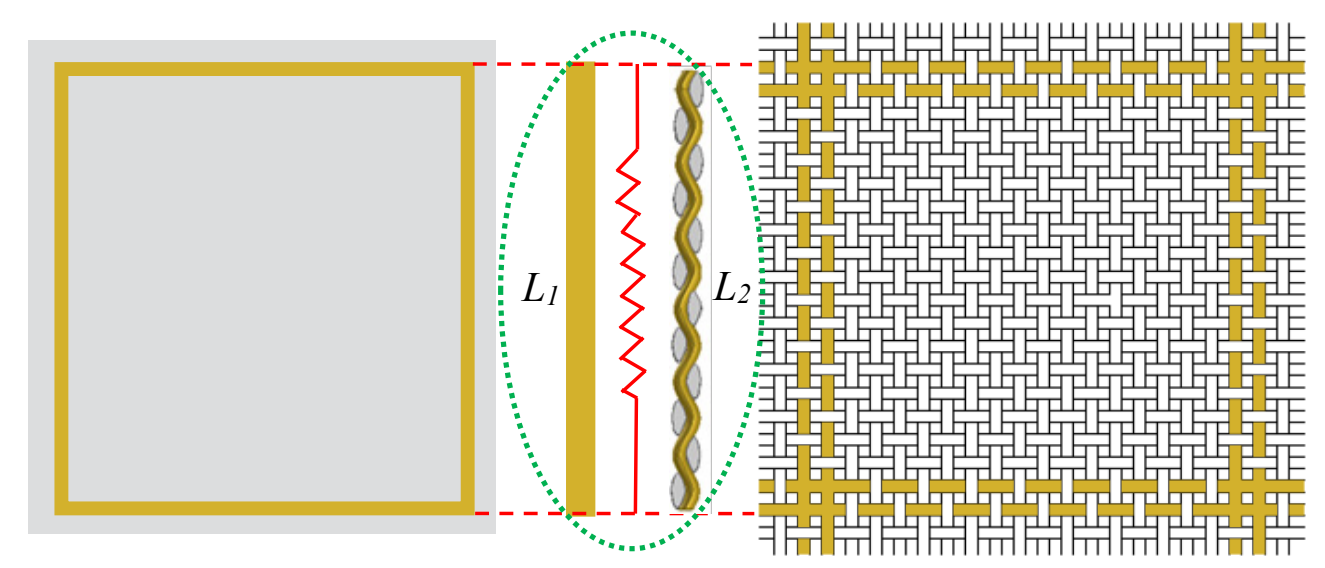

Figure 7. Unit cell comparison of two kinds of high-pass FSSs Abbreviation: FSSs, frequency selective surfaces

are same, the conductive yarn in the fabric is a bit longer in practice due to the spatial crimp, which makes the inductance of fabric unit cell slightly larger $\left(L_{2}>L_{1}\right)$ as shown in Figure 7 . Thus, more EM energy could be converted into the electron kinetic energy and less transmittance occurs at this time. In addition, more scattering effects may occur because the fabric surface is not smooth as the comparison sample and more EM waves are diffusely reflected, reducing the transmittance. The abovementioned two factors indicate that the fabric sample has stronger shielding effect and $S_{21}$ values are a little smaller correspondingly.

\subsection{Influence of polarization mode}

Due to the weaving error, the array characteristics of warp and weft directions are not completely identical, and therefore the polarization mode of EM waves would exert an influence on the transmission characteristics of fabric sample. Figure 8(a and b) shows the TE and TM transmission modes of plane EM waves, in which $\vec{E}$ and $\vec{H}$ are electric field direction and magnetic field direction, respectively; $\overrightarrow{k_{i}}, \overrightarrow{k_{t}}$, and $\overrightarrow{k_{r}}$ are the incidence wave, transmission wave, and reflection wave, respectively; $\theta_{i}$
, $\theta_{t}$, and $\theta_{r}$ are the incidence angle, transmission angle, and reflection angle, respectively; $\oplus$ and $@$ represent that the EM waves are vertically inwardly and outwardly to the XOZ plane.

As the transmission direction of EM waves is perpendicular to periodically conductive woven fabric, two transmission forms of EM waves as shown in Figure 8 could be simplified and are shown in Figure 9, in which we could see the relative position of warp and weft yarns, $\vec{E}$ and $\vec{H}$. Under the TE mode, the direction of $\vec{E}$ is along the weft yarn, and perpendicular to the warp yarn, and the contrary is the case under the TM mode.

Figure 10 shows the simulated and measured $S_{21}$ curves, from which we could see three curves, which show that the similar variation trend and specific $S_{21}$ values have little differences. During the simulation process, the geometrical and physical characteristics of warp and weft yarns are same, and also the yarn-weaving density is set to be completely identical, meaning that the array characteristics in two perpendicular directions have no differences, and that is why the simulation $S_{21}$ curves under two polarization modes are absolutely consistent. 


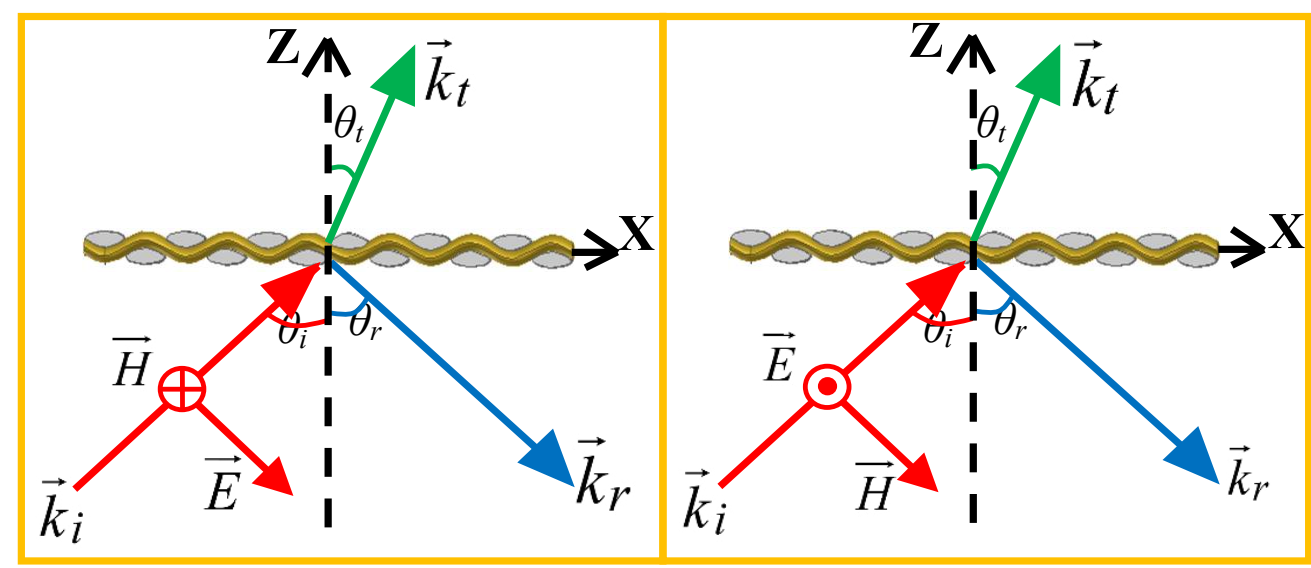

(a)

(b)

Figure 8. Two polarization modes of plane EM waves: a) TE mode; b) TM mode Abbreviations: EM, electromagnetic; TE, transverse electric; TM, transverse magnetic

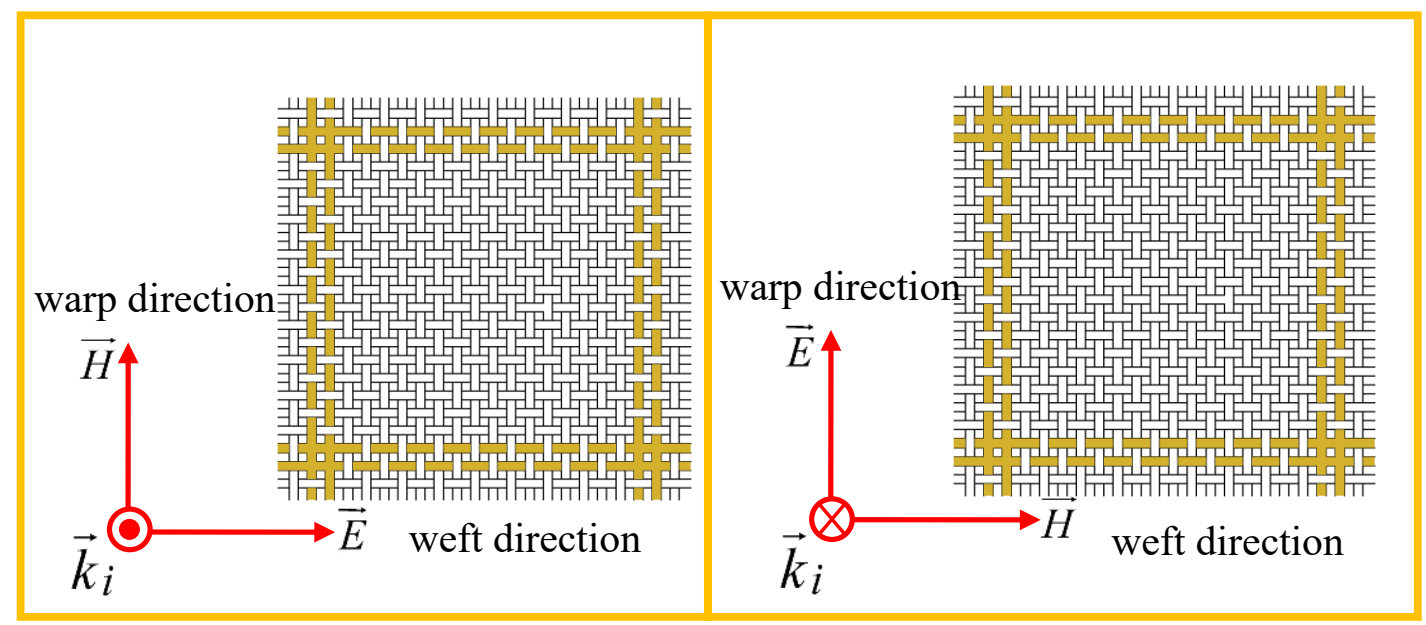

(a)

(b)

Figure 9. Relative position of warp and weft yarns, $\vec{E}$ and $\vec{H}$, as EM waves are perpendicular to periodically conductive woven fabric: a) TE mode; b) TM mode Abbreviations: EM, electromagnetic; TE, transverse electric; TM, transverse magnetic

As shown in Figure 10, the measured and simulated results have high anastomosis degree on the whole, revealing the validity of simulation process. The measurement results under two polarization modes have some differences, but the differences are small. The biggest difference appears in approximately $5.81 \mathrm{GHz}$, and the difference values are nearly $4 \mathrm{~dB}$ (from -7.44 to $-3.47 \mathrm{~dB}$ ). The reason could be attributed to the fact that experimental materials and weaving process are not exactly the same as the simulation assumptions. The actual warp density is controlled through loom reeds, and therefore it is basically consistent with the design value. However, the weft density is adjusted through beating-up tension, and therefore the tension fluctuation would have large influence, making the actual weft density not entirely uniform. The differentiated products could be easily prepared by controlling the ratio of conductive and nonconductive yarns in both directions, and the related products should be used in certain circumstances.

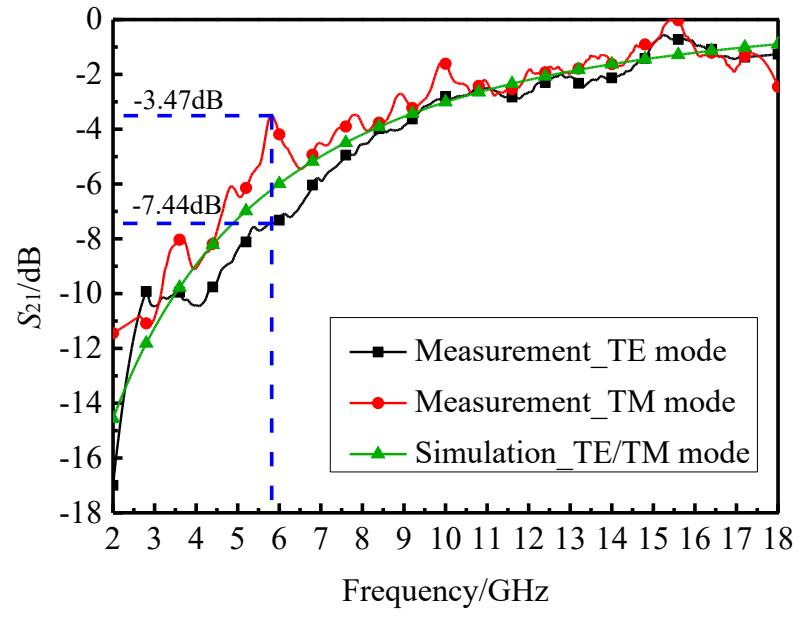

Figure 10. Comparison of simulated and measured $S_{21}$ curves $S_{21}$, transmission coefficient 


\section{CONCLUSIONS}

Based on the research status of traditional EM shielding textiles, the novel periodically conductive woven fabric with high-pass characteristics is proposed in this paper. The unit cell model was built in TexGen and simulated in CST to predict the transmission characteristics. The designed structure was fabricated using weaving process, and the sample was measured in anechoic chamber. The measured results showed good consistency with the simulated results, which verified the validity of design process. The tiny differences from the comparison sample were explored based on the specific fabric structure. In addition, the transmission characteristics under two polarization modes were compared, and the EM characteristics were indeed affected by the EM wave polarization modes, which could be attributed to the imperfection of weaving density, especially the weft density. The work could offer new research ideas for the development of EM functional textiles, and the related products are soft, lightweight, and flexible, which certainly have potential applications in certain fields, such as soft radome, radar absorbing agent, and command post tent.

\section{ACKNOWLEDGEMENT}

We greatly appreciate the financial support from Quanzhou Home-bay Recruitment Program of Global Talents (2017ZT002) and the Science and Technology Bureau of Quanzhou (2018K002).

\section{References}

[1] Sanz-Izquierdo, B., Parker, E. A., Robertson, J. B., Batchelor, J. C. (2009). Tuning technique for active FSS arrays. Electronics Letters, 45(22), 1107-1108.

[2] Haghzadeh, M., Akyurtlu, A. (2016). All-printed, flexible, reconfigurable frequency selective surfaces. Journal of Applied Physics, 120(18), 184901.

[3] Liang, B., Sanz-lzquierdo, B., Parker, E. A., Batchelor, J. C. (2015). Cylindrical slot FSS configuration for beamswitching applications. IEEE Transactions on Antennas and Propagation, 63(1), 166-173.

[4] Cheng, H. H., Xiao, H., Shi, M. W., Wang, Q., Wang, N. (2016). Research on $3 D$ periodic structure velvet fabric and its frequency response characteristics. Textile Research Journal, 86(7), 776-784.
[5] Li, B., Shen, Z. X. (2013). Angular-stable and polarizationindependent frequency selective structure with high selectivity. Applied Physics Letters, 103(17), 171607.

[6] Yun, S., Bossard, J. A., Mayer, T. S., Werner, D. H. (2010). Angle and polarization tolerant midinfrared dielectric filter designed by genetic algorithm optimization. Applied Physics Letters, 96(22), 223101.

[7] Kim, P. C., Chin, W. S., Lee, D. G., Seo, I. S. (2006). EM characteristics of the RAS composed of E-glass/epoxy composite and single dipole FSS element. Composite Structures, 75(1), 601-609.

[8] Casse, B. D. F., Moser, H. O., Jian, L. K., Bahou, M., Wilhelmi, O., et al. (2006). Fabrication of $2 D$ and $3 D$ electromagnetic metamaterials for the terahertz range. Journal of Physics: Conference Series. IOP Publishing, 885-890.

[9] Ghane, M., Ghorbani, E. (2016). Investigation into the UV-protection of woven fabrics composed of metallic weft yarns. Autex Research Journal, 16(3), 154-159.

[10] Unnikrishnan, S. K., Vinayasree, S., Halliah, G. P., Anantharaman, M. (2013). Flexible electromagnetic interference shields in $S$ band region from textile materials. Journal of Industrial Textiles, 43(2), 215-230.

[11] Saravanja, B., Malaric, K., Pusic, T., Ujevic, D. (2015). Shield effect of functional interlining fabric. Autex Research Journal, 16(3), 93-98.

[12] Guan, F. W., Xiao, H., Shi, M. W., Wang, F. M. (2016). The novel frequency selective fabric and application research. Journal of Industrial Textiles, 46(1), 143-59.

[13] Guan, F. W., Xiao, H., Shi, M. W., Yu, W. D., Wang, F. M. (2017). Realization of planar frequency selective fabrics and analysis of transmission characteristics. Textile Research Journal, 87(11), 1360-1366.

[14] Seager, R. D., Chauraya, A., Bowman, J., Broughton, M., Philpott, R., et al. (2013). Fabric based frequency selective surfaces using weaving and screen printing. Electronics Letters, 49(24), 1507-1509.

[15] Chin, K. S., Wu, C. S., Shen, C. L., Tsai, K. C. (2018). Designs of textile antenna arrays for smart clothing applications. Autex Research Journal, 16(3), 295-307.

[16] Guan, F. W., Xiao, H., Shi, M. W., Wang, F. M. (2016). The frequency response characteristics of planar frequency selective fabrics (FSFs) with cross-shaped units. Textile Research Journal, 86(20), 2169-2178.

[17] Bayatpur, F. (2009). Metamaterial-inspired frequencyselective surfaces. Doctoral thesis. The University of Michigan, 46-48.

[18] Munk, B. A. (2000). Frequency selective surfaces: theory and design. John Wiley \& Sons, Inc (New York). 